\title{
Variational Formulas for the Green Function
}

\author{
Charles Z. Martin
}

October 31, 2018

\begin{abstract}
The Green function has a complex dependence upon its underlying domain and differential operator. We briefly review Hadamard's formula for the first variation of the Green function due to a perturbation of the domain. We then take a different avenue and approximate the change in the Green function when the Laplacian is perturbed into a number of different operators: Helmholtz, Schrödinger and Laplace-Beltrami.
\end{abstract}

\section{Introduction}

The study of boundary value problems presents a significant computational challenge. In theory there are numerous formulas, transforms and methods for solving, say, the Dirichlet problem on an ellipse for the Laplace-Beltrami operator. In fact, knowledge of a single functionthe Green function-would reduce the problem to that of calculating an integral. However, the computation of a Green function is prohibitively difficult; considering its central role we might turn to methods of approximating it instead. One approach aims to replace the Green function of the problem with that of an easier problem which is, in some sense, nearby. There are two ways this heuristic can proceed: variation of the underlying domain and variation of the relevant operator.

The purpose of domain variation is clear; the Green function of a region is nearly impossible to compute for all but the nicest of domains. Approximating a given domain by a simpler one is a natural way to circumvent the difficulty. The resulting alteration to the Green function is very complicated, but a formula due to Hadamard gives the first variation, which can be sufficient information for some applications.

For other applications, we might want to approximate the Green function not under perturbations of the domain, but rather of the operator. This avenue of thought is less studied and for good reason; in many applications the differential operator is considered to be well-known. If we turn our attention to inverse problems however, we might pose questions wherein an unknown operator has a Green function with some given property. The existence of the underlying operator can be studied by understanding what sorts of variations in the Green function can result from perturbing a well-understood operator, such as the Laplacian.

In this article we briefly review a formal argument developing Hadamard's variation. With this in hand we can make a few remarks about what sorts of basic facts can follow from such a formula. From here we proceed to consider Helmholtz, Schrödinger and Laplace-Beltrami operators which are somehow close to the Laplacian. The resulting variation formulas are newor at least not widely known - and have nice forms open to interpretation, both mathematical and physical. 


\section{Definitions and Known Results}

There are numerous definitions and conventions surrounding the Green function of a domain. For clarity we take a moment to review a few important properties which will be used in the following. Let $D \subset \mathbb{C}$ be a bounded domain. A Green function $g: D \times D \rightarrow[-\infty, \infty)$ is a map satisfying three properties:

1. For every $w \in D, g(\cdot, w)$ is harmonic on $D \backslash\{w\}$ and bounded outside every neighborhood of $w$.

2. For every $w \in D$ we have $g(w, w)=-\infty$ and $g(z, w)=(2 \pi)^{-1} \log |z-w|+O(1)$ as $z \rightarrow w$.

3. For any $w \in D$ we have $\lim _{z \rightarrow \zeta} g(z, w)=0$ for quasi-every $\zeta \in \partial D$; that is, for all $\zeta$ except a set of capacity zero.

The Green function exists and is unique for any domain whose boundary has positive capacity. In particular, we will assume that $D$ has real analytic boundary. Occasionally we wish to emphasize the dependence on one argument; hence we sometimes write $g_{w}(z)$ in place of $g(z, w)$. We have the following facts:

1. For any $\zeta \in \partial D$ which possesses a barrier, $g(z, w)=0$ as $z \rightarrow \zeta$; having assumed $\partial D$ real analytic, this follows for all $\zeta$. We can thus extend $g$ to allow one of its arguments to be on $\partial D$.

2. The Green function is the solution to the boundary value problem

$$
\begin{cases}\Delta g_{w}=\delta_{w} & \text { in } D \\ g_{w}=0 & \text { on } \partial D\end{cases}
$$

It follows that the solution to a more general problem

$$
\begin{cases}\Delta u=f & \text { in } D \\ u=0 & \text { on } \partial D\end{cases}
$$

is given by integration against the Green function:

$$
u(z)=\int_{D} g_{z} f d A .
$$

This property can be taken as the definition of the Green function of $D$. We can define the Green function $g^{*}$ of an operator $L$ analogously:

$$
\begin{cases}L g_{w}^{*}=\delta_{w} & \text { in } D \\ g_{w}^{*}=0 & \text { on } \partial D\end{cases}
$$

3. The Green function is subharmonic.

4. For all $z, w \in D$ the Green function is symmetric: $g(z, w)=g(w, z)$.

5. Along $\partial D$ we have

$$
\frac{\partial g}{\partial n}(\zeta, w) d s(\zeta)=d \omega(w, \zeta)
$$

where $\omega$ represents the harmonic measure of $D$ and $\partial g / \partial n$ represents the $\zeta$ derivative in the outward normal direction. 
In what follows, we will need the Poisson-Jensen formula for subharmonic functions. Suppose $u$ is subharmonic in a neighborhood of $\bar{D}$ and $u \neq \equiv-\infty$. Then for all $z \in D$

$$
u(z)=\int_{\partial D} u(\zeta) d \omega(z, \zeta)+\int_{D} g(z, \xi) \Delta u(\xi) d A(\xi) .
$$

Even if $\Delta u$ is a distribution, the formula still holds by interpreting the area integral as a distributional pairing. Essentially this result follows from the Green identity

$$
\int_{\partial D}\left(u \frac{\partial v}{\partial n}-v \frac{\partial u}{\partial n}\right) d s=\int_{D}(u \Delta v-v \Delta u) d A
$$

with $v=g_{z}$.

\section{Domain Variation of the Green Function}

Before proceeding to variations of operators, we examine Hadamard's formula for the variation of the Green function due to change of the domain. Suppose that $D$ is slightly enlarged into a new domain $D^{*}$ in such a way so that along $\partial D$ each point is moved along the outward normal direction a small distance. The following first-order variation formula gives a relationship between the new Green function $g^{*}$ and the old one. Because this is well-known, we only give a formal argument; the original derivation can be found in 3 and a more rigorous treatment can be found in [4] or in chapter 15 of [1].

Theorem 1 (Hadamard's formula). Let $p \in C(\partial D)$ be a positive function and suppose that for $\epsilon>0$ each point $\zeta \in \partial D$ is moved along the outward normal direction a distance $\epsilon p(\zeta)$. The Green function $g^{*}$ of the new domain $D^{*}$ satisfies

$$
g^{*}(z, w)-g(z, w)=-\epsilon \int_{\partial D} \frac{\partial g}{\partial n}(z, \zeta) \frac{\partial g}{\partial n}(w, \zeta) p(\zeta) d s(\zeta)+o(\epsilon) .
$$

Proof. Fix $w \in D$ and apply the Poisson-Jensen formula to $g_{w}^{*}$ :

$$
g^{*}(z, w)=\int_{\partial D} g^{*}(\zeta, w) d \omega(z, \zeta)+\int_{D} g(z, \xi) \Delta g^{*}(\xi, w) d A(\xi) .
$$

Since $\Delta g_{w}^{*}=\delta_{w}$ and $g^{*}$ is symmetric in its arguments, this becomes

$$
g^{*}(z, w)=\int_{\partial D} g^{*}(w, \zeta) d \omega(z, \zeta)+g(z, w) .
$$

Denote the outward unit normal by $n$. For $\zeta \in \partial D, \zeta+\epsilon p(\zeta) n \in \partial D^{*}$. Therefore

$$
g^{*}(w, \zeta+\epsilon p(\zeta) n)=0 .
$$

From here we obtain the first-order approximation

$$
g^{*}(w, \zeta)+\epsilon \frac{\partial g^{*}}{\partial n}(w, \zeta) p(\zeta)+o(\epsilon)=0
$$

Using this approximation (11) becomes

$$
g^{*}(z, w)-g(z, w)=-\epsilon \int_{\partial D} \frac{\partial g}{\partial n}(z, \zeta) \frac{\partial g^{*}}{\partial n}(w, \zeta) p(\zeta) d s(\zeta)+o(\epsilon),
$$

where we have substituted $\partial g / \partial n(z, \zeta) d s(\zeta)$ for $d \omega(z, \zeta)$. Finally, we remove the dependence on $g^{*}$ by iterating the formula; that is, from (2) we have $g^{*}-g=o(1)$ and

$$
g^{*}(z, w)-g(z, w)=-\epsilon \int_{\partial D} \frac{\partial g}{\partial n}(z, \zeta) \frac{\partial}{\partial n}[g(w, \zeta)+o(1)](w, \zeta) p(\zeta) d s(\zeta)+o(\epsilon) .
$$

This gives the result. 
In traditional notation of the calculus of variations, we can write the aforementioned formula as

$$
\delta g(z, w):=\lim _{\epsilon \rightarrow 0} \frac{g^{*}(z, w)-g(z, w)}{\epsilon}=-\int_{\partial D} \frac{\partial g}{\partial n}(z, \zeta) \frac{\partial g}{\partial n}(w, \zeta) p(\zeta) d s(\zeta) .
$$

We will at times use this notation for sake of clarity.

Example 2. Consider the unit disk $\mathbb{D} \subset \mathbb{C}$. The Green function for a disk of radius $R$ is

$$
g(z, w)=\frac{1}{2 \pi} \ln \left|\frac{R(z-w)}{R^{2}-z \bar{w}}\right| .
$$

Suppose that $\mathbb{D}$ is enlarged by uniformly increasing the radius by $\epsilon$ - that is, the perturbation function $p$ is constant along $\partial D$. On one hand, a direct computation shows

$$
\delta g(z, w)=\left.\frac{\partial g}{\partial R}(z, w)\right|_{R=1}=-\frac{1}{2 \pi} \frac{1-|z w|^{2}}{|1-z \bar{w}|^{2}} .
$$

The variational formula gives

$$
\begin{aligned}
\delta g(z, w) & =-\frac{1}{2 \pi} \int_{\partial \mathbb{D}} \frac{\left(1-|w|^{2}\right)\left(1-|z|^{2}\right)}{|\zeta-w|^{2}|\zeta-z|^{2}} d s(\zeta) \\
& =-\frac{1}{2 \pi} \int_{\partial \mathbb{D}} \frac{\left(1-|w|^{2}\right)\left(1-|z|^{2}\right)}{(\zeta-w)(\zeta-z)(\bar{\zeta}-\bar{z})(\bar{\zeta}-\bar{w})} \frac{d \zeta}{i \zeta} .
\end{aligned}
$$

Since $|\zeta|=1$ we can rewrite $\bar{\zeta}-\bar{z}=(1-\zeta \bar{z}) / \zeta$. The above integral can be written as

$$
\delta g(z, w)=-\frac{1}{2 \pi i} \int_{\partial \mathbb{D}} \frac{1}{(\zeta-w)(\zeta-z)} \cdot \frac{\left(1-|w|^{2}\right)\left(1-|z|^{2}\right) \zeta}{(1-\zeta \bar{z})(1-\zeta \bar{w})} d \zeta .
$$

Note that the second factor in the integral is analytic in a neighborhood of $\overline{\mathbb{D}}$. The Cauchy integral theorem gives

$$
\begin{aligned}
\delta g(z, w) & =-\frac{1}{2 \pi i} \int_{\partial D} \frac{1}{z-w}\left(\frac{1}{\zeta-z}-\frac{1}{\zeta-w}\right) \cdot \frac{\left(1-|w|^{2}\right)\left(1-|z|^{2}\right) \zeta}{(1-\zeta \bar{z})(1-\zeta \bar{w})} d \zeta+o(\epsilon) \\
& =-\frac{1}{z-w}\left(\frac{z\left(1-|w|^{2}\right)}{1-z \bar{w}}-\frac{w\left(1-|z|^{2}\right)}{1-w \bar{z}}\right) \\
& =-\frac{1}{2 \pi} \frac{1-|z w|^{2}}{|1-z \bar{w}|^{2}}
\end{aligned}
$$

so the two answers agree.

Before proceeding we make a few remarks.

1. The outward normal derivative of the Green function is positive since, for instance, it is the density of the domain's harmonic measure. Therefore the variation is always negative, so we conclude that enlarging a domain decreases the Green function at every point.

2. Let $\lambda: \mathbb{C} \rightarrow \mathbb{R}$ be a positive smooth function and define the operator $L=\nabla \lambda \nabla$. If we alter the definition of the Green function so that $L g=\delta$, then we can derive another variational formula. From Green's identity

$$
\int_{\partial D} \lambda\left(u \frac{\partial v}{\partial n}-v \frac{\partial u}{\partial n}\right) d s=\int_{D}(u L v-v L u) d A
$$


we set $v=g_{z}$ to get an analogue of the Poisson-Jensen formula:

$$
u(z)=\int_{\partial D} u(\zeta) \lambda(\zeta) d \omega(z, \zeta)+\int_{D} g(z, \xi) \Delta u(\xi) d A(\xi) .
$$

From here we obtain the first variation of $g$.

$$
g^{*}(z, w)-g(z, w)=-\epsilon \int_{\partial D} \frac{\partial g}{\partial n}(z, \zeta) \frac{\partial g}{\partial n}(w, \zeta) \lambda(\zeta) p(\zeta) d s(\zeta)+o(\epsilon)
$$

3. In Laplacian growth dynamics, a domain containing the origin grows with outward velocity given by $V(\zeta)=\partial g / \partial n(\zeta, 0)$. In these circumstances, an infinitesimal time step $d t$ causes a domain variation with $\epsilon p=V d t$. As such, the variation formula yields

$$
\frac{d g}{d t}(z, 0)=-\int_{\partial D} \frac{\partial g}{\partial n}(z, \zeta)\left[\frac{\partial g}{\partial n}(0, \zeta)\right]^{2} d s(\zeta) .
$$

A generalization of Laplacian growth dynamics is elliptic growth dynamics, wherein we use the Green function for $L=\nabla \lambda \nabla$ and take $p(\zeta)=\lambda(\zeta) \partial g / \partial n(\zeta, 0)$. In this case the previous formula is modified into

$$
\frac{d g}{d t}(z, 0)=-\int_{\partial D} \frac{\partial g}{\partial n}(z, \zeta)\left[\lambda(\zeta) \frac{\partial g}{\partial n}(0, \zeta)\right]^{2} d s(\zeta)
$$

4. There is an alternative way of defining the Green function, wherein $g(z, w)=-\ln \mid z-$ $w \mid+O(1)$ as $z \rightarrow w$. Taking this as a definition leads to a few minor changes. Firstly, $\Delta g_{w}=-2 \pi \delta_{w}$ and the Poisson-Jensen formula reads

$$
u(z)=\int_{\partial D} u(\zeta) d \omega(z, \zeta)-\frac{1}{2 \pi} \int_{D} g(z, \xi) \Delta u(\xi) d A(\xi) .
$$

This leads to the variation formula

$$
g^{*}(z, w)-g(z, w)=\frac{\epsilon}{2 \pi} \int_{\partial D} \frac{\partial g}{\partial n}(z, \zeta) \frac{\partial g}{\partial n}(w, \zeta) p(\zeta) d s(\zeta)+o(\epsilon)
$$

\section{Operator Variation of the Green Function}

Now we turn to situations wherein the domain is fixed but rather the underlying operator is somehow close to the Laplacian. We will make repeated use of the following integral operator.

Definition 1. Given a domain $D$ define the operator $T$ to be integration against the Green function of $D$ :

$$
T \phi(z)=\int_{D} \phi(\xi) g(z, \xi) d A(\xi) \text {. }
$$

Lemma 3. The operator $T$ is a bounded linear map from $L^{2}(D)$ into $C(D)$, the space of continuous functions on $D$.

Proof. Notice that $T$ is a left inverse to the Laplacian. By elliptic regularity, $T$ maps $L^{2}$ into $H^{2}$. But in the plane, Sobolev imbedding implies that $H^{2}(D) \subset C(D)$. 


\subsection{Helmholtz}

Suppose we'd like to approximate the Green function $g^{*}$ of the Helmholtz operator $H=\Delta-a$, where $a$ is a small constant. In terms of the original Green function $g$ we can derive the following.

Theorem 4. Let $D$ be a bounded domain in $\mathbb{C}$ and fix $w \in D$. The Green function $g^{*}$ of the Helmholtz operator $\Delta-$ a satisfies

$$
g_{w}^{*}-g_{w}=a T g_{w}+o(a)
$$

as $a \rightarrow 0$, where the convergence of $o(a)$ is uniform in $z$ for each fixed $w$. Furthermore, a full series expansion is given by

$$
g_{w}^{*}=\sum_{n=0}^{\infty} a^{n} T^{n} g_{w} .
$$

Proof. The function $g_{w}^{*}$ solves the boundary value problem

$$
\left\{\begin{array}{ll}
\Delta g_{w}^{*} & =\delta_{w}+a g_{w}^{*} \\
g_{w}^{*} & =0 \quad \text { on } \partial D
\end{array} \text { in } D\right.
$$

By definition of the classical Green function, this implies that

$$
g_{w}^{*}(z)=T\left(\delta_{w}(\xi)+a g_{w}^{*}(\xi)\right)=g_{w}(z)+a T g_{w}^{*}(\xi) .
$$

If we define the error function $f=g_{w}^{*}-g_{w}$ the previous equation becomes

$$
f=a T g_{w}^{*}=a T g_{w}+a T f .
$$

It remains to show that $T f \rightarrow 0$ uniformly as $a \rightarrow 0$. For all $a$ smaller than $\|T\|^{-1}$,

$$
f=a(I-a T)^{-1} T g_{w} .
$$

Since $g_{w} \in L^{2}$ so is $(I-a T)^{-1} T g_{w}$ and we can take $a \rightarrow 0$ to find

$$
\|f\|_{2} \leq|a|\left\|(I-a T)^{-1} T g_{w}\right\|_{2} \rightarrow 0 .
$$

That is, $f \rightarrow 0$ in $L^{2}$ as $a \rightarrow 0$. The lemma implies that $T f \rightarrow 0$ in $C(D)$, hence uniformly. In addition we obtain the full series expansion

$$
g_{w}^{*}=\sum_{n=0}^{\infty} a^{n} T^{n} g_{w}
$$

if we use the Neumann series for $(I-a T)^{-1}$ in (4).

\subsection{Schrödinger}

In the previous section we deduced a perturbation formula for a Schrödinger operator with constant potential function. With slight adjustments to the proof we can handle a general potential function; the operator $\Delta-u$ also possesses a perturbation formula when $u$ is small.

Theorem 5. Let $D$ be a bounded domain in $\mathbb{C}$ and fix $w \in D$. Suppose that $p$ is a smooth scalar function defined in a neighborhood of $D$ with corresponding multiplication operator $P$. The Green function $g^{*}$ of the Schrödinger operator $\Delta-\epsilon p$ satisfies

$$
g_{w}^{*}-g_{w}=\epsilon T P g_{w}+o(\epsilon)
$$


as $\epsilon \rightarrow 0$, where the convergence of $o(\epsilon)$ is uniform in $z$ for each fixed $w$. Furthermore, a full series expansion is given by

$$
g_{w}^{*}=\sum_{n=0}^{\infty} \epsilon^{n}(T P)^{n} g_{w}
$$

Proof. The first steps of the proof are similar to the Helmholtz case. The function $g_{w}^{*}$ solves the boundary value problem

$$
\left\{\begin{array}{ll}
\Delta g_{w}^{*} & =\delta_{w}+\epsilon p g_{w}^{*} \\
g_{w}^{*} & =0 \quad \text { on } \partial D
\end{array} \text { in } D\right.
$$

By definition of the classical Green function, this implies that

$$
g_{w}^{*}=T\left(\delta_{w}+\epsilon p g_{w}^{*}\right)=g_{w}+\epsilon T P g_{w}^{*}=g_{w}+\epsilon T P g_{w}+\epsilon T P\left(g_{w}^{*}-g_{w}\right) .
$$

Define the error function $f=g_{w}^{*}-g_{w}$, so

$$
f=\epsilon T P g_{w}+\epsilon T P f .
$$

It remains to show that $\operatorname{TPf} \rightarrow 0$ uniformly as $\epsilon \rightarrow 0$.

As in our treatment of the Helmholtz operator, $T$ is a bounded linear map $L^{2} \rightarrow H^{2}$. Since $D$ is bounded, $p \in L^{\infty}(D)$; thus $P$ maps $L^{2} \rightarrow L^{2}$. Together this means that $T P$ continuously maps $L^{2} \rightarrow H^{2}$ and the rest of proof will proceed analogously as before. For all $\epsilon$ sufficiently small we find that

$$
f=\epsilon(I-\epsilon T P)^{-1} T P g_{w} .
$$

Since $g_{w} \in L^{2}$ we can take $\epsilon \rightarrow 0$ :

$$
\|f\|_{2} \leq|\epsilon|\left\|(I-\epsilon T P)^{-1} T P g_{w}\right\|_{2} \rightarrow 0 .
$$

Thus $f \rightarrow 0$ in $L^{2}$ and $T P f \rightarrow 0$ in $H^{2} \subset C(D)$. We conclude that $T f \rightarrow 0$ uniformly. The full series expansion again follows from expanding $(1-\epsilon T P)^{-1}$ in a Neumann series.

\subsection{Laplace-Beltrami}

Next we consider the problem of finding the Green function on a domain for the LaplaceBeltrami operator $L=\nabla \lambda \nabla$, where $\lambda$ is a smooth positive function. If $\lambda$ is close to unity that the Green function $g^{*}$ can be approximated by the Green function $g$ of the Laplacian. The following result makes this notion precise.

Theorem 6. Fix $w \in D$ and suppose that $p$ is a smooth scalar function in a neighborhood of $D$. Given $\epsilon>0$ we can define $\lambda(z)=1+\epsilon p(z)$. Then as $\epsilon \rightarrow 0$ the Green function $g^{*}$ for $L=\nabla \lambda \nabla$ satisfies

$$
g^{*}(z, w)-g(z, w)=\epsilon \int_{D} p(\xi) \nabla g(z, \xi) \cdot \nabla g(\xi, w) d A(\xi)+o(\epsilon),
$$

where all derivatives are with respect to $\xi$. Furthermore, the error term converges uniformly for each fixed $w$. An alternate formula is also true:

$$
g^{*}(z, w)-g(z, w)=-\epsilon g(z, w)\left(\frac{p(z)+p(w)}{2}\right)+\frac{\epsilon}{2} \int_{D} g_{z} g_{w} \Delta p d A+o(\epsilon) .
$$


Proof. We first give a heuristic argument. Once again, if we begin with the correct integration by parts formula we can formally manipulate our way to the perturbation formula above. From the Green identity

$$
\int_{\partial D} \lambda\left(u \frac{\partial v}{\partial n}-v \frac{\partial u}{\partial n}\right) d s=\int_{D}(u L v-v L u) d A
$$

we let $u=g_{z}$ and $v=g_{w}^{*}$ to get

$$
\int_{D} g_{z} L g_{w}^{*} d A=\int_{D} g_{w}^{*} L g_{z} d A
$$

Note that $L g_{w}^{*}=\delta_{w}$ and $L g_{z}=\lambda \delta_{z}+\nabla \lambda \cdot \nabla g_{z}$ to find

$$
g(z, w)=\lambda(z) g^{*}(z, w)+\int_{D} g_{w}^{*} \nabla \lambda \cdot \nabla g_{z} d A,
$$

whence

$$
\begin{aligned}
g^{*}(z, w)-g(z, w) & =-\epsilon p(z) g^{*}(z, w)-\epsilon \int_{D} g_{w}^{*} \nabla p \cdot \nabla g_{z} d A \\
& =-\epsilon p(z) g(z, w)-\epsilon \int_{D} g_{w} \nabla p \cdot \nabla g_{z} d A+\epsilon R_{\epsilon}
\end{aligned}
$$

where we have defined

$$
R_{\epsilon}=-p(z)\left(g^{*}(z, w)-g(z, w)\right)-\int_{D}\left(g_{w}^{*}-g_{w}\right) \nabla p \cdot \nabla g_{z} d A .
$$

Here we end the rigor and claim that $R_{\epsilon}=o(1)$. Notice that this is the same gap that appeared in the derivation of Hadamard's formula above. Using the claim (9) becomes

$$
g^{*}(z, w)-g(z, w)=-\epsilon p(z) g(z, w)-\epsilon \int_{D} g_{w} \nabla p \cdot \nabla g_{z} d A+o(\epsilon) .
$$

We can simplify this via integration by parts. Note that $g(\xi, w)=0$ for $\xi \in \partial D$; therefore

$$
\begin{aligned}
\int_{D} \nabla p \cdot\left(g_{w} \nabla g_{z}\right) d A & =-\int_{D} p \nabla \cdot\left(g_{w} \nabla g_{z}\right) d A \\
& =-\int_{D} p g_{w} \Delta g_{z} d A-\int_{D} p \nabla g_{z} \cdot \nabla g_{w} d A \\
& =-p(z) g(z, w)-\int_{D} p \nabla g_{z} \cdot \nabla g_{w} d A
\end{aligned}
$$

Inserting this into (10) gives our first perturbation formula (77). To deduce (8) we take (10) and reverse the roles of $z$ and $w$ :

$$
g^{*}(z, w)-g(z, w)=-\epsilon p(w) g(z, w)-\epsilon \int_{D} g_{z} \nabla p \cdot \nabla g_{w} d A+o(\epsilon) .
$$

Adding this half of this equation to half of (10), we recognize a product rule.

$$
g^{*}(z, w)-g(z, w)=-\epsilon g(z, w)\left(\frac{p(z)+p(w)}{2}\right)-\frac{\epsilon}{2} \int_{D} \nabla p \cdot \nabla\left(g_{z} g_{w}\right) d A+o(\epsilon) .
$$

Since $g(z, \xi) g(\xi, w)=0$ on $\partial D$, integrating by parts one more time gives the result.

We now proceed to derive (8) in another fashion, sidestepping the need to consider the error term $R_{\epsilon}$. Essentially we will use a change of variables to relate the problem to that of the Schödinger operator. 
Define the functions $G_{w}^{*}=g_{w}^{*} \sqrt{\lambda}$ and $u=\lambda^{-1 / 2} \Delta\left(\lambda^{1 / 2}\right)$. Notice that $G_{w}^{*}=0$ on $\partial D$ and

$$
\nabla \lambda=\nabla(\sqrt{\lambda} \sqrt{\lambda})=2 \sqrt{\lambda} \nabla \sqrt{\lambda}
$$

With this in mind we compute:

$$
\begin{aligned}
(\Delta-u) G_{w}^{*} & =\Delta\left(g_{w}^{*} \sqrt{\lambda}\right)-u g_{w}^{*} \sqrt{\lambda} \\
& =g_{w}^{*} \Delta \sqrt{\lambda}+\sqrt{\lambda} \Delta g_{w}^{*}+2 \nabla g_{w}^{*} \cdot \nabla \sqrt{\lambda}-g_{w}^{*} \Delta \sqrt{\lambda} \\
& =\frac{\lambda \Delta g_{w}^{*}+\nabla \lambda \cdot \nabla g_{w}^{*}}{\sqrt{\lambda}} \\
& =\delta_{w} / \sqrt{\lambda} \\
& =\delta_{w} / \sqrt{\lambda(w)} .
\end{aligned}
$$

From this we conclude that $\sqrt{\lambda(w)} G_{w}^{*}$ is the Green function of $D$ for the operator $\Delta-u$. We can use our perturbation formula for this operator if we can determine how $u$ depends upon $\epsilon$. We begin with a computation; from equation (11) we find that

$$
|\nabla \sqrt{\lambda}|^{2}=\left|\frac{\nabla \lambda}{2 \sqrt{\lambda}}\right|^{2}=\frac{|\nabla \lambda|^{2}}{4 \lambda} .
$$

Now we have what we need to relate $u$ and $\lambda$ without roots.

$$
\Delta \lambda=\Delta \sqrt{\lambda} \sqrt{\lambda}=2 \sqrt{\lambda} \Delta \sqrt{\lambda}+2|\nabla \sqrt{\lambda}|^{2}=2 \lambda u+\frac{|\nabla \lambda|^{2}}{2 \lambda},
$$

from which it follows that

$$
u=\frac{\Delta \lambda}{2 \lambda}-\frac{|\nabla \lambda|^{2}}{4 \lambda^{2}}=\frac{\epsilon \Delta p / 2}{1+\epsilon p}-\frac{\epsilon^{2}|\nabla p|^{2} / 4}{(1+\epsilon p)^{2}} .
$$

From here we expand in series to find

$$
\begin{aligned}
u & =\frac{\epsilon \Delta p}{2} \sum_{n=0}^{\infty}(-1)^{n}(\epsilon p)^{n}-\frac{\epsilon^{2}|\nabla p|^{2}}{4} \sum_{n=0}^{\infty} n(-1)^{n}(\epsilon p)^{n-1} \\
& =\sum_{n=0}^{\infty}(-1)^{n}\left(\frac{\epsilon \Delta p}{2}+\frac{n \epsilon|\nabla p|^{2}}{4 p}\right)(\epsilon p)^{n} \\
& =\frac{\epsilon \Delta p}{2}+o(\epsilon),
\end{aligned}
$$

where the error term is uniformly convergent for all small $\epsilon$. Therefore we have

$$
\sqrt{\lambda(w) \lambda(z)} g^{*}(z, w)=g(z, w)+\int_{D}\left(\frac{\epsilon \Delta p}{2}+o(\epsilon)\right) g_{z} g_{w} d A+o(\epsilon) .
$$

As a map $L^{\infty} \rightarrow L^{\infty}$ the integral operator

$$
\phi \mapsto \int_{D} \phi g_{z} g_{w} d A
$$

is continuous, so we deduce that

$$
\int_{D} o(\epsilon) g_{z} g_{w} d A=o(\epsilon)
$$


uniformly. We conclude that

$$
\sqrt{\lambda(w) \lambda(z)} g^{*}(z, w)=g(z, w)+\frac{\epsilon}{2} \int_{D} g_{z} g_{w} \Delta p d A+o(\epsilon) .
$$

The last step requires a first order approximation of $(\lambda(w) \lambda(z))^{-1 / 2}$ in $\epsilon$. We find that

$$
\begin{aligned}
\frac{1}{\sqrt{\lambda(w) \lambda(z)}} & =(1+\epsilon p(w))^{-1 / 2}(1+\epsilon p(z))^{-1 / 2} \\
& =\left(1-\frac{\epsilon p(z)}{2}+o(\epsilon)\right)\left(1-\frac{\epsilon p(w)}{2}+o(\epsilon)\right) \\
& =1-\epsilon\left(\frac{p(z)+p(w)}{2}\right)+o(\epsilon) .
\end{aligned}
$$

Therefore

$$
\begin{aligned}
g^{*}(z, w) & =\left[1-\epsilon\left(\frac{p(z)+p(w)}{2}\right)+o(\epsilon)\right]\left[g(z, w)+\frac{\epsilon}{2} \int_{D} g_{z} g_{w} \Delta p d A+o(\epsilon)\right] \\
& =g(z, w)-\epsilon g(z, w)\left(\frac{p(z)+p(w)}{2}\right)+\frac{\epsilon}{2} \int_{D} g_{z} g_{w} \Delta p d A+o(\epsilon),
\end{aligned}
$$

as desired.

Example 7. Again consider $\mathbb{D}$, the unit disk in $\mathbb{C}$. If we take $\lambda(\xi)=1+\epsilon|\xi|^{2}$ then

$$
\delta g(z, w)=\operatorname{Re} \frac{1}{4 \pi^{2}} \int_{\mathbb{D}}|\xi|^{2}\left(\frac{1}{\xi-w}+\frac{\bar{w}}{1-\bar{w} \xi}\right) \cdot \overline{\left(\frac{1}{\xi-z}+\frac{\bar{z}}{1-\bar{z} \xi}\right)} d A(\xi) .
$$

We can evaluate the integral as follows. First we assume that $z, w \neq 0$ and notice that

$$
\xi\left(\frac{1}{\xi-w}+\frac{\bar{w}}{1-\bar{w} \xi}\right)=\frac{w}{\xi-w}+\frac{1}{1-\bar{w} \xi},
$$

whence the integrand can be rewritten as

$$
\frac{w \bar{z}}{(\xi-w)(\bar{\xi}-\bar{z})}+\frac{w}{(1-z \bar{\xi})(\xi-w)}+\frac{\bar{z}}{(1-\xi \bar{w})(\bar{\xi}-\bar{z})}+\frac{1}{(1-\xi \bar{w})(1-\bar{\xi} z)} .
$$

We will integrate each of these four terms in turn. Following [2], we recognize the exponential transform of $\mathbb{D}$ :

$$
\begin{aligned}
\int_{\mathbb{D}} \frac{w \bar{z}}{(\xi-w)(\bar{\xi}-\bar{z})} d A(\xi) & =-\pi w \bar{z} \log E_{\mathbb{D}}(w, z) \\
& =\pi w \bar{z} \log (1-w \bar{z})-2 \pi w \bar{z} \log |z-w|
\end{aligned}
$$

Here we have used the fact that $\log (a b)=\log a+\log b(\bmod i \mathbb{R})$; ultimately we only desire the real part of each integral. This handles the first term in (12). Next consider

$$
\int_{R} \frac{w}{(1-z \bar{\xi})(\xi-w)} d A(\xi)
$$

where $R=\mathbb{D} \backslash D(w, \delta)$ and $\delta$ is a small positive number. Since $(1-z \bar{\xi})^{-1}$ is antianayltic throughout $\mathbb{D}$ we can write

$$
\begin{aligned}
\int_{R} \frac{w}{(1-z \bar{\xi})(\xi-w)} d A(\xi) & =-\frac{w}{z} \int_{R} \bar{\partial}\left(\frac{\log (1-z \bar{\xi})}{\xi-w}\right) d A(\xi) \\
& =-\frac{w}{z} \int_{\partial R} \frac{\log (1-z \bar{\xi})}{\xi-w} \frac{d \xi}{2 i}
\end{aligned}
$$


Considering orientation of the inner circle, we have

$$
-\frac{w}{z} \int_{\partial R} \frac{\log (1-z \bar{\xi})}{\xi-w} \frac{d \xi}{2 i}=-\frac{w}{z} \int_{\partial \mathbb{D}} \frac{\log (1-z \bar{\xi})}{\xi-w} \frac{d \xi}{2 i}+\frac{w}{z} \int_{\partial D(w, \delta)} \frac{\log (1-z \bar{\xi})}{\xi-w} \frac{d \xi}{2 i}
$$

To evaluate the first of these integrals we remark that $z / \xi \in \mathbb{D}$ for $\xi \in \partial \mathbb{D}$ so a series expansion gives

$$
\begin{aligned}
-\frac{w}{z} \int_{\partial \mathbb{D}} \frac{\log (1-z / \xi)}{\xi-w} \frac{d \xi}{2 i} & =\frac{\pi w}{z} \sum_{k=1}^{\infty} \int_{\partial \mathbb{D}} \frac{z^{k}}{k \xi^{k}(\xi-w)} \frac{d \xi}{2 \pi i} \\
& =\frac{\pi w}{z} \sum_{j, k=1}^{\infty} \int_{\partial \mathbb{D}} \frac{z^{k} w^{j-1}}{k \xi^{k+j}} \frac{d \xi}{2 \pi i} \\
& =0 .
\end{aligned}
$$

The dominated convergence theorem implies that

$$
\begin{aligned}
\lim _{\delta \rightarrow 0} \frac{w}{z} \int_{\partial D(w, \delta)} \frac{\log (1-z \bar{\xi})}{\xi-w} \frac{d \xi}{2 i} & =\lim _{\delta \rightarrow 0} \frac{w}{z} \int_{0}^{2 \pi} \log \left(1-z \bar{w}-\delta z e^{-i \theta}\right) \frac{d \theta}{2} \\
& =\frac{\pi w}{z} \log (1-z \bar{w})
\end{aligned}
$$

Thus

$$
\int_{\mathbb{D}} \frac{w}{(1-z \bar{\xi})(\xi-w)} d A(\xi)=\frac{\pi w}{z} \log (1-z \bar{w})
$$

Similarly,

$$
\int_{\mathbb{D}} \frac{\bar{z}}{(1-\xi \bar{w})(\bar{\xi}-\bar{z})} d A(\xi)=\frac{\pi \bar{z}}{\bar{w}} \log (1-z \bar{w}) .
$$

The last term in (12) can be evaluated as such:

$$
\begin{aligned}
\int_{\mathbb{D}} \frac{1}{(1-\xi \bar{w})(1-\bar{\xi} z)} d A(\xi) & =-\frac{1}{z} \int_{\mathbb{D}} \bar{\partial}\left(\frac{\log (1-z \bar{\xi})}{1-\xi \bar{w}}\right) d A(\xi) \\
& =-\frac{1}{z} \int_{\partial \mathbb{D}} \frac{\log (1-z / \xi)}{1-\xi \bar{w}} \frac{d \xi}{2 i} \\
& =\sum_{j, k=1}^{\infty} \int_{\partial \mathbb{D}} \frac{z^{k-1} \bar{w}^{j-1}}{k \xi^{k-j+1}} \frac{d \xi}{2 i} \\
& =\pi \sum_{k=1}^{\infty} \frac{(z \bar{w})^{k-1}}{k} \\
& =-\frac{\pi}{z \bar{w}} \log (1-z \bar{w}) .
\end{aligned}
$$

Altogether we have

$$
\delta g(z, w)=\frac{1}{4 \pi} \operatorname{Re}\left[\left(z \bar{w}+\frac{\bar{z}}{\bar{w}}+\frac{w}{z}-\frac{1}{z \bar{w}}\right) \log (1-z \bar{w})-2 z \bar{w} \log |z-w|\right],
$$

where we have taken complex conjugates of various terms to combine them better. Note that this formula only depends upon rotationally-invariant quantities such as $z \bar{w}$, as can be expected.

Next we assume that $w=0$ and compute

$$
\begin{aligned}
\delta g(z, 0) & =\operatorname{Re} \frac{1}{4 \pi^{2}} \int_{\mathbb{D}}|\xi|^{2} \bar{\xi}^{-1} \cdot\left(\frac{1}{\xi-z}+\frac{\bar{z}}{1-\bar{z} \xi}\right) d A(\xi) \\
& =\operatorname{Re} \frac{1}{4 \pi^{2}} \int_{\mathbb{D}}\left(\frac{\xi}{\xi-z}+\frac{\bar{z} \xi}{1-\bar{z} \xi}\right) d A(\xi) .
\end{aligned}
$$


The second term is a harmonic function in $\xi$; therefore we have

$$
\int_{\mathbb{D}} \frac{\bar{z} \xi}{1-\bar{z} \xi} d A(\xi)=\left.\pi \frac{\bar{z} \xi}{1-\bar{z} \xi}\right|_{\xi=0}=0 .
$$

Given a small $\delta>0$ define $D_{\delta}=\mathbb{D} \backslash D(z, \delta)$ and use Stokes' theorem to write

$$
\begin{aligned}
\int_{D_{\delta}} \frac{\xi}{\xi-z} d A(\xi) & =\int_{D_{\delta}} \bar{\partial}\left(\frac{|\xi|^{2}}{\xi-z}\right) d A(\xi) \\
& =\int_{\partial \mathbb{D}} \frac{|\xi|^{2}}{\xi-z} \frac{d \xi}{2 i}-\int_{\partial D(z, \delta)} \frac{|\xi|^{2}}{\xi-z} \frac{d \xi}{2 i} \\
& =\pi-\frac{1}{2} \int_{0}^{2 \pi}\left|z+\delta e^{i \theta}\right|^{2} d \theta \\
& \rightarrow \pi\left(1-|z|^{2}\right)
\end{aligned}
$$

as $\delta \rightarrow 0$. From this we conclude that

$$
\delta g(z, 0)=\frac{1-|z|^{2}}{4 \pi} .
$$

We remark the same result is obtained by taking $w \rightarrow 0$ in equation (13).

\section{References}

[1] P. R. Garabedian, Partial Differential Equations, Chelsea, New York 1986.

[2] B. Gustafsson, M. Putinar, An exponential transform and regularity of free boundaries in two dimensions, Ann. Scuola Norm. Sup. Pisa Cl. Sci. (4), 26 (1998), 507-543.

[3] J. Hadamard, Mémoire sur le problème d'analyse relatif à l'équilibre des plaques élastiques encastrées, Mémoires presentés par divers savants à l'Académie des Sciences 33 (1908), $1-128$.

[4] E. Schippers, W. Staubach, Variation of Neumann and Green functions under homotopies of the boundary, Israel J. Math. 173 (2009), 279-303.

Department of Mathematics, University of California, Santa Barbara, CA 93106

E-mail: cmart07@math.ucsb.edu 